\title{
Unsupervised Relation Disambiguation Using Spectral Clustering
}

\author{
Jinxiu Chen ${ }^{1}$ \\ ${ }^{1}$ Institute for Infocomm Research \\ 21 Heng Mui Keng Terrace \\ 119613 Singapore \\ \{jinxiu, dhji, zniu\}@i2r.a-star.edu.sg
}

\author{
Chew Lim Tan ${ }^{2} \quad$ Zhengyu Niu ${ }^{1}$ \\ ${ }^{2}$ Department of Computer Science \\ National University of Singapore \\ 117543 Singapore \\ tanclecomp.nus.edu.sg
}

\begin{abstract}
This paper presents an unsupervised learning approach to disambiguate various relations between name entities by use of various lexical and syntactic features from the contexts. It works by calculating eigenvectors of an adjacency graph's Laplacian to recover a submanifold of data from a high dimensionality space and then performing cluster number estimation on the eigenvectors. Experiment results on ACE corpora show that this spectral clustering based approach outperforms the other clustering methods.
\end{abstract}

\section{Introduction}

In this paper, we address the task of relation extraction, which is to find relationships between name entities in a given context. Many methods have been proposed to deal with this task, including supervised learning algorithms (Miller et al., 2000; Zelenko et al., 2002; Culotta and Soresen, 2004; Kambhatla, 2004; Zhou et al., 2005), semi-supervised learning algorithms (Brin, 1998; Agichtein and Gravano, 2000; Zhang, 2004), and unsupervised learning algorithm (Hasegawa et al., 2004).

Among these methods, supervised learning is usually more preferred when a large amount of labeled training data is available. However, it is time-consuming and labor-intensive to manually tag a large amount of training data. Semi-supervised learning methods have been put forward to minimize the corpus annotation requirement. Most of semi-supervised methods employ the bootstrapping framework, which only need to pre-define some initial seeds for any particular relation, and then bootstrap from the seeds to acquire the relation. However, it is often quite difficult to enumerate all class labels in the initial seeds and decide an "optimal" number of them.

Compared with supervised and semi-supervised methods, Hasegawa et al. (2004)'s unsupervised approach for relation extraction can overcome the difficulties on requirement of a large amount of labeled data and enumeration of all class labels. Hasegawa et al. (2004)'s method is to use a hierarchical clustering method to cluster pairs of named entities according to the similarity of context words intervening between the named entities. However, the drawback of hierarchical clustering is that it required providing cluster number by users. Furthermore, clustering is performed in original high dimensional space, which may induce non-convex clusters hard to identified.

This paper presents a novel application of spectral clustering technique to unsupervised relation extraction problem. It works by calculating eigenvectors of an adjacency graph's Laplacian to recover a submanifold of data from a high dimensional space, and then performing cluster number estimation on a transformed space defined by the first few eigenvectors. This method may help us find non-convex clusters. It also does not need to pre-define the number of the context clusters or pre-specify the similarity threshold for the clusters as Hasegawa et al. (2004)'s method.

The rest of this paper is organized as follows. Section 2 formulates unsupervised relation extraction and presents how to apply the spectral clustering 
technique to resolve the task. Then section 3 reports experiments and results. Finally we will give a conclusion about our work in section 4 .

\section{Unsupervised Relation Extraction Problem}

Assume that two occurrences of entity pairs with similar contexts, are tend to hold the same relation type. Thus unsupervised relation extraction problem can be formulated as partitioning collections of entity pairs into clusters according to the similarity of contexts, with each cluster containing only entity pairs labeled by the same relation type. And then, in each cluster, the most representative words are identified from the contexts of entity pairs to induce the label of relation type. Here, we only focus on the clustering subtask and do not address the relation type labeling subtask.

In the next subsections we will describe our proposed method for unsupervised relation extraction, which includes: 1) Collect the context vectors in which the entity mention pairs co-occur; 2) Cluster these Context vectors.

\subsection{Context Vector and Feature Design}

Let $X=\left\{x_{i}\right\}_{i=1}^{n}$ be the set of context vectors of occurrences of all entity mention pairs, where $x_{i}$ represents the context vector of the $i$-th occurrence, and $n$ is the total number of occurrences of all entity mention pairs.

Each occurrence of entity mention pairs can be denoted as follows:

$$
R \rightarrow\left(C_{\text {pre }}, e_{1}, C_{\text {mid }}, e_{2}, C_{\text {post }}\right)
$$

where $e_{1}$ and $e_{2}$ represents the entity mentions, and $C_{\text {pre }}, C_{m i d}$, and $C_{\text {post }}$ are the contexts before, between and after the entity mention pairs respectively.

We extracted features from $e_{1}, e_{2}, C_{\text {pre }}, C_{m i d}$, $C_{\text {post }}$ to construct context vectors, which are computed from the parse trees derived from Charniak Parser (Charniak, 1999) and the Chunklink script ${ }^{1}$ written by Sabine Buchholz from Tilburg University.

Words: Words in the two entities and three context windows.

\footnotetext{
${ }^{1}$ Software available at http://ilk.uvt.nl/ sabine/chunklink/
}

Entity Type: the entity type of both entities, which can be PERSON, ORGANIZATION, FACILITY, LOCATION and GPE.

POS features: Part-Of-Speech tags corresponding to all words in the two entities and three context windows.

Chunking features: This category of features are extracted from the chunklink representation, which includes:

- Chunk tag information of the two entities and three context windows. The " 0 " tag means that the word is outside of any chunk. The "I-XP" tag means that this word is inside an XP chunk. The "B-XP" by default means that the word is at the beginning of an XP chunk.

- Grammatical function of the two entities and three context windows. The last word in each chunk is its head, and the function of the head is the function of the whole chunk. "NP-SBJ" means a NP chunk as the subject of the sentence. The other words in a chunk that are not the head have "NOFUNC" as their function.

- IOB-chains of the heads of the two entities. So-called IOB-chain, noting the syntactic categories of all the constituents on the path from the root node to this leaf node of tree.

We combine the above lexical and syntactic features with their position information in the context to form the context vector. Before that, we filter out low frequency features which appeared only once in the entire set.

\subsection{Context Clustering}

Once the context vectors of entity pairs are prepared, we come to the second stage of our method: cluster these context vectors automatically.

In recent years, spectral clustering technique has received more and more attention as a powerful approach to a range of clustering problems. Among the efforts on spectral clustering techniques (Weiss, 1999; Kannan et al., 2000; Shi et al., 2000; Ng et al., 2001; Zha et al., 2001), we adopt a modified version 
Table 1: Context Clustering with Spectral-based Clustering technique.

Input: A set of context vectors $X=\left\{x_{1}, x_{2}, \ldots, x_{n}\right\}$, $X \in \Re^{n \times d}$

Output: Clustered data and number of clusters;

1. Construct an affinity matrix by $A_{i j}=\exp \left(-\frac{s_{i j}^{2}}{\sigma^{2}}\right)$ if $i \neq$ $j, 0$ if $i=j$. Here, $s_{i j}$ is the similarity between $x_{i}$ and $x_{j}$ calculated by Cosine similarity measure. and the free distance parameter $\sigma^{2}$ is used to scale the weights;

2. Normalize the affinity matrix $A$ to create the matrix $L=$ $D^{-1 / 2} A D^{-1 / 2}$, where $D$ is a diagonal matrix whose $(i, i)$ element is the sum of $A$ 's $i$ th row;

3. Set $q=2$;

4. Compute $q$ eigenvectors of $L$ with greatest eigenvalues. Arrange them in a matrix $Y$.

5. Perform elongated $K$-means with $q+1$ centers on $Y$, initializing the $(q+1)$-th mean in the origin;

6. If the $q+1$-th cluster contains any data points, then there must be at least an extra cluster; set $q=q+1$ and go back to step 4. Otherwise, algorithm stops and outputs clustered data and number of clusters.

(Sanguinetti et al., 2005) of the algorithm by $\mathrm{Ng}$ et al. (2001) because it can provide us model order selection capability.

Since we do not know how many relation types in advance and do not have any labeled relation training examples at hand, the problem of model order selection arises, i.e. estimating the "optimal" number of clusters. Formally, let $k$ be the model order, we need to find $k$ in Equation: $k=$ $\operatorname{argmax}_{k}\{\operatorname{criterion}(k)\}$. Here, the criterion is defined on the result of spectral clustering.

Table 1 shows the details of the whole algorithm for context clustering, which contains two main stages: 1) Transformation of Clustering Space (Step 1-4); 2) Clustering in the transformed space using Elongated K-means algorithm (Step 5-6).

\subsection{Transformation of Clustering Space}

We represent each context vector of entity pair as a node in an undirected graph. Each edge $(i, j)$ in the graph is assigned a weight that reflects the similarity between two context vectors $i$ and $j$. Hence, the relation extraction task for entity pairs can be defined as a partition of the graph so that entity pairs that are more similar to each other, e.g. labeled by the same relation type, belong to the same cluster. As a relaxation of such NP-hard discrete graph partitioning problem, spectral clustering technique computes eigenvalues and eigenvectors of a Laplacian matrix related to the given graph, and construct data clusters based on such spectral information.

Thus the starting point of context clustering is to construct an affinity matrix $A$ from the data, which is an $n \times n$ matrix encoding the distances between the various points. The affinity matrix is then normalized to form a matrix $L$ by conjugating with the the diagonal matrix $D^{-1 / 2}$ which has as entries the square roots of the sum of the rows of $A$. This is to take into account the different spread of the various clusters (points belonging to more rarified clusters will have lower sums of the corresponding row of $A$ ). It is straightforward to prove that $L$ is positive definite and has eigenvalues smaller or equal to 1 , with equality holding in at least one case.

Let $K$ be the true number of clusters present in the dataset. If $K$ is known beforehand, the first $K$ eigenvectors of $L$ will be computed and arranged as columns in a matrix $Y$. Each row of $Y$ corresponds to a context vector of entity pair, and the above process can be considered as transforming the original context vectors in a $d$-dimensional space to new context vectors in the $K$-dimensional space. Therefore, the rows of $Y$ will cluster upon mutually orthogonal points on the $K$ dimensional sphere,rather than on the coordinate axes.

\subsection{The Elongated K-means algorithm}

As the step 5 of Table 1 shows, the result of elongated $K$-means algorithm is used to detect whether the number of clusters selected $q$ is less than the true number $K$, and allows one to iteratively obtain the number of clusters.

Consider the case when the number of clusters $q$ is less than the true cluster number $K$ present in the dataset. In such situation, taking the first $q<K$ eigenvectors, we will be selecting a $q$-dimensional subspace in the clustering space. As the rows of the $K$ eigenvectors clustered along mutually orthogonal vectors, their projections in a lower dimensional space will cluster along radial directions. Therefore, the general picture will be of $q$ clusters elongated in the radial direction, with possibly some clusters very near the origin (when the subspace is orthogonal to some of the discarded eigenvectors).

Hence, the $K$-means algorithm is modified as the elongated $K$-means algorithm to downweight distances along radial directions and penalize dis- 


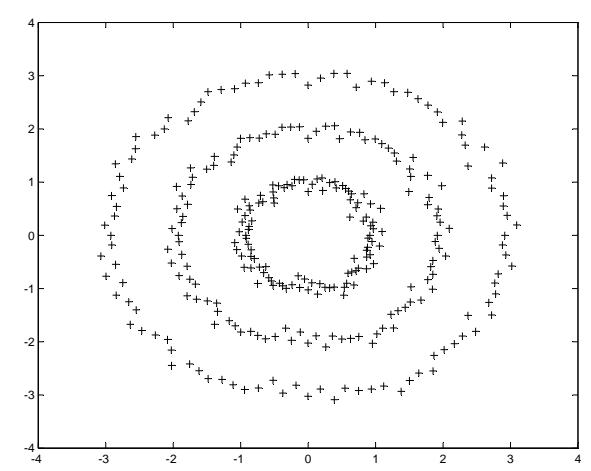

(a)

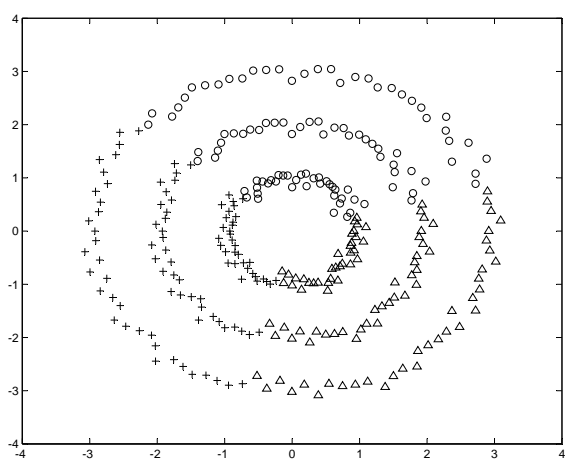

(b)
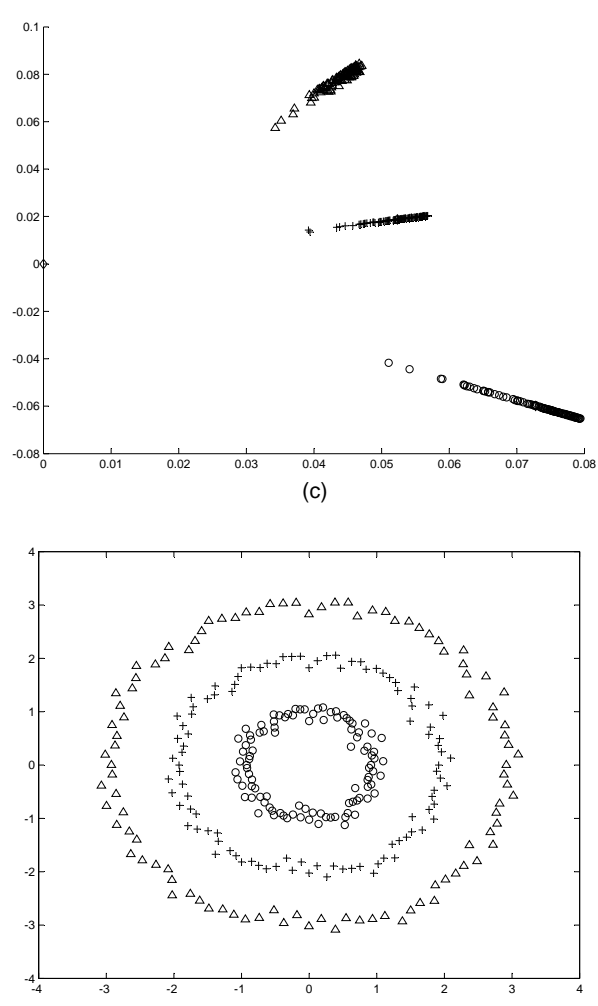

(d)

Figure 1: An Example:(a) The Three Circle Dataset. (b) The clustering result using K-means; (c) Three elongated clusters in the 2D clustering space using Spectral clustering: two dominant eigenvectors; (d) The clustering result using Spectral-based clustering $\left(\sigma^{2}=0.05\right) .(\triangle, \circ$ and + denote examples in different clusters) tances along transversal directions. The elongated $K$-means algorithm computes the distance of point $\mathrm{x}$ from the center $c_{i}$ as follows:

- If the center is not very near the origin, $c_{i}^{T} c_{i}>\epsilon(\epsilon$ is a parameter to be fixed by the user), the distances are calculated as: $\operatorname{edist}\left(x, c_{i}\right)=\left(x-c_{i}\right)^{T} M\left(x-c_{i}\right)$, where $M=\frac{1}{\lambda}\left(I_{q}-\frac{c_{i} c_{i}^{T}}{c_{i}^{T} c_{i}}\right)+\lambda \frac{c_{i} c_{i}^{T}}{c_{i}^{T} c_{i}}, \lambda$ is the sharpness parameter that controls the elongation (the smaller, the more elongated the clusters) ${ }^{2}$.

- If the center is very near the origin, $c_{i}^{T} c_{i}<\epsilon$, the distances are measured using the Euclidean distance.

In each iteration of procedure in Table 1, elongated $K$-means is initialized with $q$ centers corresponding to data points in different clusters and one center in the origin. The algorithm then will drag the center in the origin towards one of the clusters not accounted for. Compute another eigenvector (thus increasing the dimension of the clustering space to $q+1$ ) and repeat the procedure. Eventually, when one reach as many eigenvectors as the number of clusters present in the data, no points will be assigned to the center at the origin, leaving the cluster empty. This is the signal to terminate the algorithm.

\subsection{An example}

Figure 1 visualized the clustering result of three circle dataset using K-means and Spectral-based clustering. From Figure 1(b), we can see that K-means can not separate the non-convex clusters in three circle dataset successfully since it is prone to local minimal. For spectral-based clustering, as the algorithm described, initially, we took the two eigenvectors of $L$ with largest eigenvalues, which gave us a twodimensional clustering space. Then to ensure that the two centers are initialized in different clusters, one center is set as the point that is the farthest from the origin, while the other is set as the point that simultaneously farthest the first center and the origin. Figure 1(c) shows the three elongated clusters in the 2D clustering space and the corresponding clustering result of dataset is visualized in Figure 1(d), which exploits manifold structure (cluster structure) in data.

\footnotetext{
${ }^{2}$ In this paper, the sharpness parameter $\lambda$ is set to 0.2
} 
Table 2: Frequency of Major Relation SubTypes in the ACE training and devtest corpus.

\begin{tabular}{l|l|rr}
\hline Type & SubType & Training & Devtest \\
\hline \hline ROLE & General-Staff & 550 & 149 \\
& Management & 677 & 122 \\
& Citizen-Of & 127 & 24 \\
& Founder & 11 & 5 \\
& Owner & 146 & 15 \\
& Affiliate-Partner & 111 & 15 \\
& Member & 460 & 145 \\
& Client & 67 & 13 \\
& Other & 15 & 7 \\
\hline PART & Part-Of & 490 & 103 \\
& Subsidiary & 85 & 19 \\
& Other & 2 & 1 \\
\hline AT & Located & 975 & 192 \\
& Based-In & 187 & 64 \\
& Residence & 154 & 54 \\
\hline SOC & Other-Professional & 195 & 25 \\
& Other-Personal & 60 & 10 \\
& Parent & 68 & 24 \\
& Spouse & 21 & 4 \\
& Associate & 49 & 7 \\
& Other-Relative & 23 & 10 \\
& Sibling & 7 & 4 \\
& GrandParent & 6 & 1 \\
\hline NEAR & Relative-Location & 88 & 32 \\
\hline
\end{tabular}

\section{Experiments and Results}

\subsection{Data Setting}

Our proposed unsupervised relation extraction is evaluated on ACE 2003 corpus, which contains 519 files from sources including broadcast, newswire, and newspaper. We only deal with intra-sentence explicit relations and assumed that all entities have been detected beforehand in the EDT sub-task of ACE. To verify our proposed method, we only collect those pairs of entity mentions which have been tagged relation types in the given corpus. Then the relation type tags were removed to test the unsupervised relation disambiguation. During the evaluation procedure, the relation type tags were used as ground truth classes. A break-down of the data by 24 relation subtypes is given in Table 2 .

\subsection{Evaluation method for clustering result}

When assessing the agreement between clustering result and manually annotated relation types (ground truth classes), we would encounter the problem that there was no relation type tags for each cluster in our clustering results.

To resolve the problem, we construct a contin- gency table $T$, where each entry $t_{i, j}$ gives the number of the instances that belong to both the $i$-th estimated cluster and $j$-th ground truth class. Moreover, to ensure that any two clusters do not share the same labels of relation types, we adopt a permutation procedure to find an one-to-one mapping function $\Omega$ from the ground truth classes (relation types) $T C$ to the estimated clustering result $E C$. There are at most $|T C|$ clusters which are assigned relation type tags. And if the number of the estimated clusters is less than the number of the ground truth clusters, empty clusters should be added so that $|E C|=|T C|$ and the one-to-one mapping can be performed, which can be formulated as the function: $\hat{\Omega}=\arg \max _{\Omega} \sum_{j=1}^{|T C|} t_{\Omega(j), j}$, where $\Omega(j)$ is the index of the estimated cluster associated with the $j$-th class.

Given the result of one-to-one mapping, we adopt Precision, Recall and F-measure to evaluate the clustering result.

\subsection{Experimental Design}

We perform our unsupervised relation extraction on the devtest set of ACE corpus and evaluate the algorithm on relation subtype level. Firstly, we observe the influence of various variables, including Distance Parameter $\sigma^{2}$, Different Features, Context Window Size. Secondly, to verify the effectiveness of our method, we further compare it with other two unsupervised methods.

\subsubsection{Choice of Distance Parameter $\sigma^{2}$}

We simply search over $\sigma^{2}$ and pick the value that finds the best aligned set of clusters on the transformed space. Here, the scattering criterion trace $\left(P_{W}^{-1} P_{B}\right)$ is used to compare the cluster quality for different value of $\sigma^{2}{ }^{3}$, which measures the ratio of between-cluster to within-cluster scatter. The higher the $\operatorname{trace}\left(P_{W}^{-1} P_{B}\right)$, the higher the cluster quality.

In Table 3 and Table 4, with different settings of feature set and context window size, we find out the

\footnotetext{
${ }^{3} \operatorname{trace}\left(P_{W}^{-1} P_{B}\right)$ is trace of a matrix which is the sum of its diagonal elements. $P_{W}$ is the within-cluster scatter matrix as: $P_{W}=\sum_{j=1}^{c} \sum_{X_{i} \in \chi_{j}}\left(X_{i}-m_{j}\right)\left(X_{i}-m_{j}\right)^{t}$ and $P_{B}$ is the between-cluster scatter matrix as: $P_{B}=\sum_{j=1}^{c}\left(m_{j}-\right.$ $m)\left(m_{j}-m\right)^{t}$, where $\mathrm{m}$ is the total mean vector and $m_{j}$ is the mean vector for $j^{\text {th }}$ cluster and $\left(X_{j}-m_{j}\right)^{t}$ is the matrix transpose of the column vector $\left(X_{j}-m_{j}\right)$.
} 
Table 3: Contribution of Different Features

\begin{tabular}{l|rcc|rrr}
\hline Features & $\sigma^{2}$ & cluster number & trace value & Precison & Recall & F-measure \\
\hline \hline Words & 0.021 & 15 & 2.369 & $41.6 \%$ & $30.2 \%$ & $34.9 \%$ \\
+Entity Type & 0.016 & 18 & 3.198 & $40.3 \%$ & $42.5 \%$ & $41.5 \%$ \\
+POS & 0.017 & 18 & 3.206 & $37.8 \%$ & $46.9 \%$ & $41.8 \%$ \\
+Chunking Infomation & 0.015 & 19 & 3.900 & $43.5 \%$ & $49.4 \%$ & $46.3 \%$ \\
\hline
\end{tabular}

Table 4: Different Context Window Size Setting

\begin{tabular}{c|rcc|rrr}
\hline Context Window Size & $\sigma^{2}$ & cluster number & trace value & Precision & Recall & F-measure \\
\hline \hline 0 & 0.016 & 18 & 3.576 & $37.6 \%$ & $48.1 \%$ & $42.2 \%$ \\
2 & 0.015 & 19 & 3.900 & $43.5 \%$ & $49.4 \%$ & $46.3 \%$ \\
5 & 0.020 & 21 & 2.225 & $29.3 \%$ & $34.7 \%$ & $31.7 \%$ \\
\hline
\end{tabular}

corresponding value of $\sigma^{2}$ and cluster number which maximize the trace value in searching for a range of value $\sigma^{2}$.

\subsubsection{Contribution of Different Features}

As the previous section presented, we incorporate various lexical and syntactic features to extract relation. To measure the contribution of different features, we report the performance by gradually increasing the feature set, as Table 3 shows.

Table 3 shows that all of the four categories of features contribute to the improvement of performance more or less. Firstly,the addition of entity type feature is very useful, which improves $F$-measure by $6.6 \%$. Secondly, adding POS features can increase $F$-measure score but do not improve very much. Thirdly, chunking features also show their great usefulness with increasing Precision/Recall/F-measure by $5.7 \% / 2.5 \% / 4.5 \%$.

We combine all these features to do all other evaluations in our experiments.

\subsubsection{Setting of Context Window Size}

We have mentioned in Section 2 that the context vectors of entity pairs are derived from the contexts before, between and after the entity mention pairs. Hence, we have to specify the three context window size first. In this paper, we set the mid-context window as everything between the two entity mentions. For the pre- and post- context windows, we could have different choices. For example, if we specify the outer context window size as 2 , then it means that the pre-context (post-context)) includes two words before (after) the first (second) entity.

For comparison of the effect of the outer context of entity mention pairs, we conducted three different
Table 5: Performance of our proposed method (Spectralbased clustering) compared with other unsupervised methods: ((Hasegawa et al., 2004))'s clustering method and K-means clustering.

\begin{tabular}{l|rrl}
\hline & Precision & Recall & F-measure \\
\hline \hline Hasegawa's Method1 & $38.7 \%$ & $29.8 \%$ & $33.7 \%$ \\
Hasegawa's Method2 & $37.9 \%$ & $36.0 \%$ & $36.9 \%$ \\
Kmeans & $34.3 \%$ & $40.2 \%$ & $36.8 \%$ \\
Our Proposed Method & $43.5 \%$ & $49.4 \%$ & $46.3 \%$ \\
\hline
\end{tabular}

settings of context window size $(0,2,5)$ as Table 4 shows. From this table we can find that with the context window size setting, 2 , the algorithm achieves the best performance of $43.5 \% / 49.4 \% / 46.3 \%$ in Precision/Recall/F-measure. With the context window size setting, 5 , the performance becomes worse because extending the context too much may include more features, but at the same time, the noise also increases.

\subsubsection{Comparison with other Unsupervised methods}

In (Hasegawa et al., 2004), they preformed unsupervised relation extraction based on hierarchical clustering and they only used word features between entity mention pairs to construct context vectors. We reported the clustering results using the same clustering strategy as Hasegawa et al. (2004) proposed. In Table 5, Hasegawa's Method1 means the test used the word feature as Hasegawa et al. (2004) while Hasegawa's Method2 means the test used the same feature set as our method. In both tests, we specified the cluster number as the number of ground truth classes.

We also approached the relation extraction problem using the standard clustering technique, K- 
means, where we adopted the same feature set defined in our proposed method to cluster the context vectors of entity mention pairs and pre-specified the cluster number as the number of ground truth classes.

Table 5 reports the performance of our proposed method comparing with the other two unsupervised methods. Table 5 shows our proposed spectral based method clearly outperforms the other two unsupervised methods by $12.5 \%$ and $9.5 \%$ in $F$-measure respectively. Moreover, the incorporation of various lexical and syntactic features into Hasegawa et al. (2004)'s method2 makes it outperform Hasegawa et al. (2004)'s method1 which only uses word feature.

\subsection{Discussion}

In this paper, we have shown that the modified spectral clustering technique, with various lexical and syntactic features derived from the context of entity pairs, performed well on the unsupervised relation extraction problem. Our experiments show that by the choice of the distance parameter $\sigma^{2}$, we can estimate the cluster number which provides the tightest clusters. We notice that the estimated cluster number is less than the number of ground truth classes in most cases. The reason for this phenomenon may be that some relation types can not be easily distinguished using the context information only. For example, the relation subtypes "Located", "Based-In" and "Residence" are difficult to disambiguate even for human experts to differentiate.

The results also show that various lexical and syntactic features contain useful information for the task. Especially, although we did not concern the dependency tree and full parse tree information as other supervised methods (Miller et al., 2000; $\mathrm{Cu}$ lotta and Soresen, 2004; Kambhatla, 2004; Zhou et al., 2005), the incorporation of simple features, such as words and chunking information, still can provide complement information for capturing the characteristics of entity pairs. This perhaps dues to the fact that two entity mentions are close to each other in most of relations defined in ACE. Another observation from the result is that extending the outer context window of entity mention pairs too much may not improve the performance since the process may incorporate more noise information and affect the clustering result.
As regards the clustering technique, the spectralbased clustering performs better than direct clustering, K-means. Since the spectral-based algorithm works in a transformed space of low dimensionality, data can be easily clustered so that the algorithm can be implemented with better efficiency and speed. And the performance using spectralbased clustering can be improved due to the reason that spectral-based clustering overcomes the drawback of K-means (prone to local minima) and may find non-convex clusters consistent with human intuition.

Generally, from the point of view of unsupervised resolution for relation extraction, our approach already achieves best performance of 43.5\%/49.4\%/46.3\% in Precision/Recall/F-measure compared with other clustering methods.

\section{Conclusion and Future work}

In this paper, we approach unsupervised relation extraction problem by using spectral-based clustering technique with diverse lexical and syntactic features derived from context. The advantage of our method is that it doesn't need any manually labeled relation instances, and pre-definition the number of the context clusters. Experiment results on the ACE corpus show that our method achieves better performance than other unsupervised methods, i.e.Hasegawa et al. (2004)'s method and Kmeans-based method.

Currently we combine various lexical and syntactic features to construct context vectors for clustering. In the future we will further explore other semantic information to assist the relation extraction problem. Moreover, instead of cosine similarity measure to calculate the distance between context vectors, we will try other distributional similarity measures to see whether the performance of relation extraction can be improved. In addition, if we can find an effective unsupervised way to filter out unrelated entity pairs in advance, it would make our proposed method more practical.

\section{References}

Agichtein E. and Gravano L.. 2000. Snowball: Extracting Relations from large Plain-Text Collections, In Proc. of the $5^{\text {th }}$ ACM International Conference on Digital Libraries (ACMDL'OO). 
Brin Sergey. 1998. Extracting patterns and relations from world wide web. In Proc. of WebDB Workshop at 6th International Conference on Extending Database Technology (WebDB'98). pages 172-183.

Charniak E.. 1999. A Maximum-entropy-inspired parser. Technical Report CS-99-12.. Computer Science Department, Brown University.

Culotta A. and Soresen J. 2004. Dependency tree kernels for relation extraction, In proceedings of 42th Annual Meeting of the Association for Computational Linguistics. 21-26 July 2004. Barcelona, Spain.

Defense Advanced Research Projects Agency. 1995. Proceedings of the Sixth Message Understanding Conference (MUC-6) Morgan Kaufmann Publishers, Inc.

Hasegawa Takaaki, Sekine Satoshi and Grishman Ralph 2004. Discovering Relations among Named Entities from Large Corpora, Proceeding of Conference ACL2004. Barcelona, Spain.

Kambhatla N. 2004. Combining lexical, syntactic and semantic features with Maximum Entropy Models for extracting relations, In proceedings of 42th Annual Meeting of the Association for Computational Linguistics. 21-26 July 2004. Barcelona, Spain.

Kannan R., Vempala S., and Vetta A.. 2000. On clustering: Good,bad and spectral. In Proceedings of the 41st Foundations of Computer Science. pages 367-380.

Miller S.,Fox H.,Ramshaw L. and Weischedel R. 2000. A novel use of statistical parsing to extract information from text. In proceedings of 6th Applied Natural Language Processing Conference. 29 April-4 may 2000, Seattle USA.

Ng Andrew.Y, Jordan M., and Weiss Y.. 2001. On spectral clustering: Analysis and an algorithm. In Proceedings of Advances in Neural Information Processing Systems. pages 849-856.

Sanguinetti G., Laidler J. and Lawrence N.. 2005. Automatic determination of the number of clusters using spectral algorithms.In: IEEE Machine Learning for Signal Processing. 28-30 Sept 2005, Mystic, Connecticut, USA.

Shi J. and Malik.J. 2000. Normalized cuts and image segmentation. IEEE Transactions on Pattern Analysis and Machine Intelligence. 22(8):888-905.

Weiss Yair. 1999. Segmentation using eigenvectors: A unifying view. ICCV(2). pp.975-982.

Zelenko D., Aone C. and Richardella A.. 2002. Kernel Methods for Relation Extraction, Proceedings of the Conference on Empirical Methods in Natural Language Processing (EMNLP). Philadelphia.
Zha H.,Ding C.,Gu.M,He X., and Simon H.. 2001. Spectral Relaxation for $k$-means clustering. In Neural Information Processing Systems (NIPS2001). pages 1057-1064, 2001

Zhang Zhu. 2004. Weakly-supervised relation classification for Information Extraction, In proceedings of ACM 13th conference on Information and Knowledge Management (CIKM'2004). 8-13 Nov 2004. Washington D.C.,USA.

Zhou GuoDong, Su Jian, Zhang Jie and Zhang min. 2005. Exploring Various Knowledge in Relation Extraction, In proceedings of 43th Annual Meeting of the Association for Computational Linguistics. USA. 